EPiC Series in Computing
Volume 69, 2020, Pages 180-186
Proceedings of 35th International Confer-
ence on Computers and Their Applications

\title{
Predicting Cerebral Aneurysm Rupture by Gradient Boosting Decision Tree using Clinical, Hemodynamic, and Morphological Information
}

\author{
Toshiyuki Haruhara ${ }^{1}$, Hideto Ohgi ${ }^{1}$, Masaaki Suzuki ${ }^{1}$, Hiroyuki Takao ${ }^{23}$, \\ Takashi Suzuki ${ }^{2}$, Soichiro Fujimura ${ }^{23}$, Toshihiro Ishibashi ${ }^{2}$, Makoto Yamamoto ${ }^{3}$, \\ Yuichi Murayama ${ }^{2}$, and Hayato Ohwada ${ }^{1}$ \\ 1 Tokyo University of Science, Chiba, Japan \\ 7419526@ed.tus.ac.jp, 7415020@ed.tus.ac.jp, m-suzuki@rs.tus.ac.jp, ohwada@rs.tus.ac.jp \\ 2 Jikei University School of Medicine, Tokyo, Japan \\ takao@jikei.ac.jp, shittakarevolution@gmail.com, s.fujimura5016@gmail.com, \\ t-ishibashi@jikei.ac.jp, ymurayama@jikei.ac.jp \\ 3 Tokyo University of Science, Tokyo, Japan \\ yamamoto@rs.kagu.tus.ac.jp
}

\begin{abstract}
Stroke is a serious cerebrovascular condition in which brain cells die due to an abrupt blockage of arteries supplying blood and oxygen or when a blood vessel bursts or ruptures and causes bleeding in the brain. Because the onset of stroke is very sudden in most people, prevention is often difficult. In Japan, stroke is one of the major causes of death and is associated with high medical costs; these problems are exacerbated by the aging population. Therefore, stroke prediction and treatment are important. The incidence of stroke may be avoided by preventive treatment based on the patient's risk of stroke. However, since judging the risk of stroke onset is largely dependent upon the individual experience and skill of the doctor, a highly accurate prediction method that is independent of the doctor's experience and skills is necessary. This study focuses on a predictive method for subarachnoid hemorrhage, which is a type of stroke. LightGBM was used to predict the rupture of cerebral aneurysms using a machine learning model that takes clinical, hemodynamic and morphological information into account. This model was used to analyze samples from 338 cerebral aneurysm cases (35 ruptured, 303 unruptured). Simulation of cerebral blood-flow was used to calculate the hemodynamic features while the surface curvature was extracted from the 3D blood-vessel-shape data as morphological features. This model yielded a sensitivity of 0.77 and a specificity of 0.83 .
\end{abstract}

\section{Introduction}

Stroke is a generic term that encompasses cerebral infarction, cerebral hemorrhage, and subarachnoid hemorrhage and occurs is when brain cells die due to an abrupt blockage of arteries that supply blood and oxygen to the brain or bleeding in the brain tissue when a blood vessel 
bursts. For many people, stroke may occur suddenly and without warning; thus, it is difficult to prevent. In 2018, stroke became the country's fourth leading cause of death due to illness and the number one cause of being bedridden in Japan. Therefore, early prediction and treatment options for stroke patients are crucial. Reducing the incidence of stroke requires a preventive strategy that lowers the risk of stroke. Unfortunately, evaluating the risk of stroke largely depends on the individual judgment and expertise of the doctor. Therefore, a highly accurate method for predicting stroke risk that is independent of the doctor's experience and judgment is required.

Existing stroke-prediction models [11], [12] have incorporated features that are clinically verified or have been manually selected by medical experts. [8], [10] and [20] used data from the patient's medical history as input features in their research. Meanwhile, Amini et al. [2] used the k-nearest neighbor's algorithm [1] and the C4.5 decision tree method [16] for predicting stroke onset from the patient's medical history data. Moreover, some studies have started employing vascular imaging for predicting disease onset. For example, Nogueira et al. [14] employed vascular imaging to predict clinical outcomes and investigated the risk of symptomatic intracerebral hemorrhage among patients who underwent intravenous thrombolytic treatment. On the other hand, Bentley et al. [3] used computerized tomography brain-image inputs into a support vector machine (SVM) algorithm [7] to predict stroke.

There are several other reports wherein the state of cerebral blood flow, in addition to the patient's medical information, was deeply involved with the stroke onset [5]. Morino et al. [13] used particle image velocimetry (PIV) and laser doppler velocimetry (LDV) to measure the velocity profiles of ruptured and unruptured intra-aneurysmal hemodynamics. Xiang et al. [21] examined how an inlet waveform affects the predicted hemodynamics in patient-specific aneurysm geometries. Furthermore, several groups acknowledged the importance of wall shear stress (WSS), energy loss (EL), and pressure loss coefficient (PLC) in predicting the rupture of cerebral aneurysms [15], [17], [19].

Among these studies, very few have considered combining data from various technological sources to successfully predict the onset of stroke. In this regard, our previous study combined clinical information, hemodynamic information, and morphological information into a classification model for enhanced prediction of stroke [18]. Moreover, Suzuki et al. [18] aimed to develop a highly precise stroke-onset prediction method using machine learning. Specifically, they developed a machine learning model that would predict whether a cerebral aneurysm would rupture and cause subsequent subarachnoid hemorrhage using clinical information, hemodynamic information obtained by computational fluid dynamics (CFD) simulation data of cerebral blood flow, and morphological information obtained from the 3D blood-vessel-shape data as inputs. Using logistic regression as a classification model, Suzuki et al. [18] found that this model yielded a sensitivity of 0.64 and a specificity of 0.85 .

In this paper, LightGBM [9], which is a gradient-boosting algorithm based on the decision tree model, is used as a classifier. In this classifier, the time-series data obtained from the CFD simulation of cerebral blood flow were additionally considered as hemodynamic features. Additionally, the surface curvature data showing the cerebral aneurysm that was obtained from the $3 \mathrm{D}$ blood vessel shape data were considered as morphological features.

In this manuscript, we describe the data required to build the proposed classification model (Section 2), the process of building the classifier (Section 3), and the results of the numerical experiments as well as the implications of these results (Section 4). We conclude the paper in Section 5. 


\section{Dataset}

Out of the 6,470 total cases that were previously registered in the Jikei University database, we first extracted cases based on the location of the occurrence of the aneurysm. If the case was unruptured, we then extracted the cases that are being observed and have not been treated in the past. If the case was ruptured, we then extracted the cases that ruptured during followup visits. In addition, we used morphological classification to restrict the cases to those in which the length, width, and neck of the bulge are each $<10 \mathrm{~mm}$ but at least one of these measurements was $>3 \mathrm{~mm}$. Furthermore, we restricted the unruptured cases to those in which the follow-up period ${ }^{1}$ was over two years and analyzed all consecutive cases. In the end, 338 cases were selected for this study. Clinical, hemodynamic, and morphological information was extracted from the 338 cases, which included 303 unruptured and 35 ruptured samples.

\subsection{Clinical information}

The following clinical information was obtained for each case: patient age; gender; the location of the aneurysm; a patient history of subarachnoid hemorrhage (SAH); a history of smoking; diabetes mellitus (DM); hypertension (HT); hyperlipidemia; alcohol consumption; polycystic kidneys (PK); cerebral hemorrhage $(\mathrm{CH})$; hormone replacement $(\mathrm{HR})$; the date of last consultation (discretized in units of three months and in units of ten days.); family history of SAH (FH_SAH); family history of unruptured aneurysms (FH_Unruptured Aneurysm); and a family history of PK (FH_PK). A total of 32 features were collected from the patients' medical history.

\subsection{Hemodynamic Information}

Hemodynamic information was obtained through the CFD simulation of the cerebral blood flow. CFD is a branch of fluid mechanics that employs numerical analyses to solve problems involving fluid dynamics. The simulation identified physical blood-flow characteristics such as PLC, EL, energy loss per unit volume (ELV), inflow concentration index (ICI), WSS, oscillatory shear index (OSI), low shear-stress area percentage (LSA), low shear index (LSI), and shear concentration index (SCI). While our previous study [18] used only the maximum, minimum, amplitude, and average of these quantities, the maximum value : minimum value ratios of the PLC, EL, ELV, ICI, LSA, LSI, and SCI were also used in this paper. Among these characteristics, PLC, EL and WSS were reported as being helpful for predicting whether a cerebral aneurysm would rupture [15], [17], [19]. In addition, we extracted time-series features of cerebral blood flow velocity, pressure, shear force, and WSS from the CFD simulation data. The length of the time-series data of velocity, pressure, shear force, and WSS obtained from the CFD simulation was 0.80 seconds while the sampling interval was 0.05 seconds. From the inside of the cerebral aneurysm, the positions where the value of each physical quantity took the maximum value during 0.8 seconds and the positions where the variance of the values of each physical quantity took the maximum value during 0.8 seconds (eight positions in total) were found. Next, the rates of change during time window of 0.05 seconds for each physical quantity at those positions were used for time-series features for the machine learning model. A total of 181 features were collected from the blood-flow-simulation data.

The calculation conditions are summarized below. A prototype CFD solver (Siemens Healthcare GmbH, Forchheim, Germany, "Not to be used for Diagnosis and/or Therapy"), which utilizes the Lattice Boltzmann method [4], was used for this method. With regards to the physical

\footnotetext{
${ }^{1}$ The follow-up period is defined as the time between the initial consultation and the final consultation.
} 
properties of blood, the fixed density and viscosity values were set and non-Newtonian fluids were disregarded. After considering the laminar flow field, the two pulses were calculated using the pulse conditions and only the results obtained from the second pulse were used. The outlet boundary condition was set to an average static pressure of $0 \mathrm{~Pa}$, and the calculations were established in a structured computational grid with a maximum size of $0.1 \mathrm{~mm}$. Further details are described in previously published studies [15],[19].

\subsection{Morphological Information}

The morphological information of cerebral aneurysm that was obtained includes the maximum aneurysm height, maximum neck diameter, neck area, volume, aspect ratio, sidewall or bifurcation type, and the presence or absence of a bleb. To extract additional features from the 3D blood vessel shape data, which was stored in the stereolithography (STL) format, this study estimated curvatures on the surface of the vessel and used these characteristics as features. This method yielded the following four characteristics related to surface curvature: mean curvature, Gaussian curvature, root mean square (RMS) curvature, and absolute curvature. MeshLab [6] was used to read and analyze the STL files of blood-vessel-shape and obtain the surface curvatures. We used the histogram of each of four surface curvature as morphological features. A total of 257 features were collected from the morphological data.

\section{Classification Model for Cerebral Aneurysm Rupture Prediction}

LightGBM, which is an open-source software library, was used as a classifier to predict whether a cerebral aneurysm would rupture. LightGBM provides a gradient-boosting decision tree framework. Gradient boosting is a type of ensemble learning where multiple models ("weak learners") are trained to solve the same problem and combined to obtain better predictive performance. Boosting trains weak learners sequentially based on the previous weak learners. LightGBM is one of the most popular methods that is used in data analysis competitions due to its high efficiency and predictive power.

LightGBM internally produces predicted probability values ranging between 0.0 and 1.0 rather than predicted label values such as rupture or unruptured. Therefore, we need to set a probability threshold to label the outcome to be ruptured or unruptured. To determine the threshold value, the harmonic mean of the sensitivity and specificity was used for the threshold evaluation. The sensitivity, which was computed using Eq. (1), represents the fraction of ruptured samples that were correctly predicted out of the total number of ruptured samples.

$$
\text { Sensitivity }=\frac{\text { TruePositive }}{\text { TruePositive }+ \text { FalseNegative }}
$$

The specificity, which was computed by Eq. (2), represents the fraction of correctly-predicted unruptured samples out of the total number of unruptured samples.

$$
\text { Specificity }=\frac{\text { TrueNegative }}{\text { TrueNegative }+ \text { FalsePositive }}
$$

A threshold value that maximizes the harmonic mean of sensitivity and specificity was regarded as the optimal threshold value $\left(T_{\mathrm{opt}}\right)$. We calculated the harmonic mean, $H$, using the following 
equation:

$$
H=\frac{2 \cdot \text { Sensitivity } \cdot \text { Specificity }}{\text { Sensitivity }+ \text { Specificity }}
$$

To improve the sensitivity of the classifier, fine-tuning was performed by multiplying the obtained optimum threshold, $T_{\mathrm{opt}}$, by 0.9 using the equation below.

$$
T_{\mathrm{opt}}^{*}=0.9 \cdot T_{\mathrm{opt}}
$$

Other hyperparameters were tuned manually.

\section{Results and Discussion}

\subsection{Hyperparameter Tuning}

The result of the hyperparameter tuning are organized in Table 1. The default values were used for the other hyperparameters.

\begin{tabular}{lr}
\hline \hline Hyperparamater & Selected value \\
\hline \hline objective & binary \\
n_estimators & 10 \\
learning_rate & 0.02 \\
max_depth & 6 \\
\hline \hline
\end{tabular}

Table 1: Hyperparameters selected

\subsection{Predicting Cerebral Aneurysm Rupture}

The classification model was evaluated by its sensitivity, specificity, and F-measure. The Fmeasure is the harmonic mean of precision and sensitivity and it was computed using Eq. (6).

$$
\text { F-measure }=\frac{2 \cdot \text { TruePositive }}{2 \cdot \text { TruePositive }+ \text { FalsePositive }+ \text { FalseNegative }}
$$

Stratified tenfold cross-validation was used to test the performance of the classification model. Table 2 shows the confusion matrix and Table 3 summarizes the performance measures resulting from the classification of the test data. By using a gradient-boosting decision tree framework and newly added features, the sensitivity of the model was greatly improved. Therefore, the classification was more stable compared to our previous study, which yielded a sensitivity of 0.64 and specificity of 0.85 [18].

\begin{tabular}{llcc}
\hline \hline $\mathrm{N}=338$ & \multicolumn{3}{c}{ Actual class } \\
\hline & Rupture & Unrupture \\
\hline Predicted & Rupture & 27 & 53 \\
class & Unrupture & 8 & 250 \\
\hline \hline
\end{tabular}

Table 2: Confusion matrix 


\begin{tabular}{lr}
\hline Performance measure & Value \\
\hline Sensitivity & 0.77 \\
Specificity & 0.83 \\
F-measure & 0.47 \\
\hline
\end{tabular}

Table 3: Performance measures resulting from the classification

\section{Conclusions}

A classifier incorporating clinical, hemodynamic and morphological data was constructed using machine learning and used to predict cerebral aneurysm rupture in a total of 338 cerebral aneurysm data samples (35 ruptured, 303 unruptured). Using LightGBM as a classification model, we created a model with a sensitivity of 0.77 and a specificity of 0.83 that predicted cerebral aneurysm rupture using data from three different sources. Future studies will include evaluating the contribution of each parameter to the prediction performance and systematically executing the tuning of hyperparameters.

\section{Acknowledgments}

This paper is based on results obtained from a project commissioned by the New Energy and Industrial Technology Development Organization (NEDO). CFD calculations were performed in collaboration with Siemens Healthcare based on a collaboration agreement with Jikei University.

\section{References}

[1] N.S. Altman. An introduction to kernel and nearest-neighbor nonparametric regression. The American Statistician, 46(3):175-185, 1992.

[2] L. Amini, R. Azarpazhouh, M.T. Farzadfar, S.A. Mousavi, F. Jazaieri, F. Khorvash, R. Norouzi, and N. Toghianfar. Prediction and control of stroke by data mining. Int J Prev Med., 4(Suppl 2):S245-249, 2013.

[3] P. Bentley, J. Ganesalingam, A.L.C. Jones, K. Mahady, S. Epton, P. Rinne, P. Sharma, O. Halse, A. Mehta, and D. Rueckert. Prediction of stroke thrombolysis outcome using ct brain machine learning. Neuroimage Clin., 30(4):635-640, 2014.

[4] S. Chen and G.D. Doolen. Lattice boltzmann method for fluid flows. Annual Review of Fluid Mechanics, 30(1):329-364, 1998.

[5] B. Chung and J.R. Cebral. Cfd for evaluation and treatment planning of aneurysms: review of proposed clinical uses and their challenges. Ann Biomed Eng., 43(1):122-138, 2015.

[6] P. Cignoni, M. Callieri, M. Corsini, M. Dellepiane, F. Ganovelli, and G. Ranzuglia. Meshlab: an open-source mesh processing tool. Proc. of the 6th Eurographics Italian Chapter Conference, pages 129-136, 2008.

[7] C. Cortes and V.N. Vapnik. Support-vector networks. Machine Learning, 20(3):273-297, 1995.

[8] G.A. Hitman, H. Colhoun, C. Newman, M. Szarek, D.J. Betteridge, P.N. Durrington, J. Fuller, S. Livingstone, H.A. Neil, and CARDS Investigators. Stroke prediction and stroke prevention with atorvastatin in the collaborative atorvastatin diabetes study (cards). Diabet Med., 24(12):13131321, 2007.

[9] G. Ke, Q. Meng, T. Finley, T. Wang, W. Chen, W. Ma, Q. Ye, and T-Y. Liu. Lightgbm: A highly efficient gradient boosting decision tree. Advances in Neural Information Processing Systems, 30:3149-3157, 2017. 
[10] B. Letham, C. Rudin, T.H. McCormick, and D. Madigan. Stroke prediction and stroke prevention with atorvastatin in the collaborative atorvastatin diabetes study (cards). Annals of Applied Statistics, 9(3):1350-1371, 2015.

[11] T. Lumley, R.A. Kronmal, M. Cushman, T.A. Manolio, and S. Goldstein. A stroke prediction score in the elderly: validation and web-based application. J Clin Epidemiol, 55(2):129-136, 2002.

[12] T.A. Manolio, R.A. Kronmal, G.L. Burke, D.H. O'Leary, and T.R. Price. Short-term predictors of incident stroke in older adults. Stroke, 27(9):1479-1486, 1996.

[13] T. Morino, T. Tanoue, S. Tateshima, F. Vinuela, and K. Tanishita. Intra-aneurysmal blood flow based on patient-specific ct angiogram. Experiments in Fluids, 49(2):485-496, 2010.

[14] R.C. Nogueira, E. Bor-Seng-Shu, N.P. Saeed, M.J. Teixeira, R.B. Panerai, and T.G. Robinson. Meta-analysis of vascular imaging features to predict outcome following intravenous rtpa for acute ischemic stroke. Frontiers in Neurology, 7(77):1-8, 2016.

[15] Y. Qian, H. Takao, M. Umezu, and Y. Murayama. Risk analysis of unruptured aneurysms using computational fluid dynamics technology: preliminary results. AJNR Am J Neuroradiol., 32(10):1948-1955, 2011.

[16] J.R. Quinlan. C4.5: Programs for Machine Learning. Morgan Kaufmann Publishers, 1993.

[17] M. Shojima, M. Oshima, K. Takagi, R. Torii, M. Hayakawa, K. Katada, A. Morita, and T. Kirino. Magnitude and role of wall shear stress on cerebral aneurysm: computational fluid dynamic study of 20 middle cerebral artery aneurysms. Stroke, 35(11):2500-2505, 2004.

[18] M. Suzuki, T. Haruhara, H. Takao, T. Suzuki, S. Fujimura, T. Ishibashi, M. Yamamoto, Y. Murayama, and $\mathrm{H}$. Ohwada. Classification model for cerebral aneurysm rupture prediction using medical and blood-flow-simulation data. Proc. of the 11th International Conference on Agents and Artificial Intelligence, 2:895-899, 2019.

[19] H. Takao, Y. Murayama, T. Abe, T. Ishibashi, I. Yuki, S. Otsuka, T. Suzuki, S. Masuda, A. Mohamed, I. Sen, M. Yamamoto, and T. Abe. Cfd reveals hemodynamic differences between unruptured and ruptured intracranial aneurysms during observation. Stroke, 43(2):A2731, 2012.

[20] T.J. Wang, J.M. Massaro, D. Levy, R.S. Vasan, P.A. Wolf, R.B. D’Agostino, M.G. Larson, W.B. Kannel, and E.J. Benjamin. A risk score for predicting stroke or death in individuals with newonset atrial fibrillation in the community: the framingham heart study. JAMA, 290(8):1049-1056, 2003.

[21] J. Xiang, A.H. Siddiqui, and H. Meng. The effect of inlet waveforms on computational hemodynamics of patient-specific intracranial aneurysms. J Biomech., 47(16):3882-3890, 2014. 